\title{
Heart failure with preserved ejection fraction in elderly. From pathophysiology to treatment: An unresolved problem
}

\author{
Matteo Beltrami, Carlo Fumagalli \\ Cardio-Thoracic and Vascular Department, University of Florence, Italy
}

\begin{abstract}
Heart failure with preserved ejection fraction (HFpEF) has a significant impact on healthcare resources and while its occurrence in the elderly is increasing, its prognosis has not improved. Despite the prevalence of $\mathrm{HFpEF}$, the understanding of its pathophysiology is still incomplete, and optimal treatment remains largely undefined. The net clinical benefit of medical treatment with ACE inhibitors, aldosterone receptor blockers (ARBs), mineralcorticoid receptor antagonists (MRAs) and beta-blockers has led to the incorporation of these drugs into HF clinical practice guidelines. However, little or no progress has been done for patients with $\mathrm{HFpEF}$ and there are no convincing and validated therapies able to reduce mortality or morbidity. $\mathrm{HFpEF}$ is a heterogeneous clinical syndrome embracing varieties of phenotypes and could benefit from a phenotype-specific approach. In the era of precision medicine, targeted approaches have proved effective in various disciplinary medical settings and for this reason this modern approach should be encouraged also in cardiology. In elderly patients, multilevel strategies and interventions aimed at improving adherence to guidelines and tailoring therapy, could be the key to improving outcome, and to reducing costs related to HF-related re-admissions. In the present review we briefly discuss current information available regarding pathophysiology, outcome, treatment and safety of the most common drugs used in this "geriatric syndrome".
\end{abstract}

\footnotetext{
Correspondence: Matteo Beltrami, Cardio-Thoracic and Vascular Department, University of Florence, Italy.

Tel. +39.577.48982.

E-mail: beltrami.matteo1@gmail.com
}

Key words: Heart failure with preserved ejection fraction; treatment; pathophysiology; outcome; beta-blockers; angiotensin-converting enzyme inhibitor.

Conflict of Interest: The Authors declare no conflict of interest.

Received for publication: 3 January 2019.

Accepted for publication: 20 May 2019.

CCopyright M. Beltrami and C. Fumagalli, 2019

Licensee PAGEPress, Italy

Monaldi Archives for Chest Disease 2019; 89:1026

doi: 10.4081/monaldi.2019.1026

This article is distributed under the terms of the Creative Commons Attribution Noncommercial License (by-nc 4.0) which permits any noncommercial use, distribution, and reproduction in any medium, provided the original author(s) and source are credited.

\section{HFpEF syndrome from pathophysiology to outcome}

Heart failure with preserved ejection fraction (HFpEF) is a complex clinical syndrome in which patients have symptoms and signs of heart failure (HF) with normal or near normal left ventricular ejection fraction (LVEF). Its diagnosis in the elderly is challenging due to the presence of atypical symptoms related to aging and potential cardiovascular and non-cardiovascular comorbidities.

Mortality, morbidity and hospitalization for HF represent a public health dilemma worldwide; in particular short-term hospital readmission for HF occurs within 6 months. The HF syndrome shows a significant impact on the health-care resources and its occurrence is ever increasing in the elderly [1]; among older women, $>80 \%$ of new cases of HF are HFpEF [2]. HFpEF in elderly patients is a systemic syndrome where advanced age, comorbidities with organ system deterioration, frailty and impaired cognition significantly impact outcome. After hospitalization for acute HF, these co-factors often remain unaddressed thus resulting in higher healthcare systems costs, prolonged physical disability, poor quality-of-life, exercise intolerance and finally with higher rehospitalization rates and mortality [3].

There are a number of potential mechanisms that may trigger and sustain HFpEF. Diastolic dysfunction is regarded as the principal actor but several age- and sex-related modifications in the cardiac structure and function are also present. For example, older women seem to show different heart rate and stroke volume responses to exercise if compared to men; in addition, in female patients, the left ventricle (LV) response to chronic systolic hypertension is impaired LV diastolic function. Conversely, male patients' response to pressure overload is more frequently a LV dilatation with thin walls and a depressed LVEF. Other pathophysiologic factors may contribute to HFpEF syndrome, such as impaired arterial stiffening and myocardial stiffness associated with an abnormal diastolic relaxation.

In fact, reduced vasodilation properties and increased vascular stiffness, together with systemic inflammation, are well established triggers of myocardial microvascular endothelial activation with the expression of adhesion molecules (e.g., ICAM, E-selectin, etc.). Overall, these processes lead to an increased vascular pressure and fibrosis, that are altogether transmitted backward to large vessels and myocardial cells. Last but not least, in older age, reduced myocardial and vascular responsiveness to $\beta$-adrenergic stimulation is present together with coronary flow reserve impairment and decreased mitochondrial adenosine triphosphate (ATP) production in response to increased energetic demand [4].

In clinical practice, a specific diagnostic algorithm applicable for early HFpEF recognition is not available yet. Symptom- 
wise, the one most common indicator of HFpEF is exertional dyspnoea, characterized by exertional fatigue and intolerance to physical activity.

The diagnosis is generally made by patient's history collection and physical examination, echocardiography and doppler studies, and, when necessary, third level exams such as cardiac catheterization. As of today, the majorities of HFpEF studies measured the diastolic function only at rest rather than during exercise where symptoms become manifest [5].

In addition, recently, microRNAs, small non-coding RNA molecules that regulate gene expression, were shown to be involved as putative post-transcriptional pathophysiological contributors to HFpEF and to have biomarker potential such that could be used as patient pheno-groupers $[6,7]$.

\section{The limbo area of heart failure with preserved ejection fraction: Is there any effective therapy?}

While knowledge of heart failure with reduced ejection fraction (HFrEF) therapy has improved, little or no progress has been done for patients with HFpEF and there is a general consensus of lacking data to support any specific treatment for this condition. In particular the traditional drugs used in HF failed to demonstrate a morbidity and mortality reduction in HFpEF [8]. The findings of the systematic review and meta-analysis of Zheng et al. [9] display a reduction in all-cause and cardiovascular mortality with beta-blockers therapy ( $22 \%$ and $25 \%$, respectively) compared with placebo in patients with HFpEF. On the contrary, therapeutic drugs such as ACEi, aldosterone receptor blockers (ARBs) and mineralocorticoid receptor antagonists (MRAs) have not been associated with a reduction of cardiovascular events during the follow up period [9]. In several trials, therapies targeting the renin-angiotensin system have failed to find a beneficial effect in HFpEF in terms of overall mortality reduction [10,11]. In particular, the CHARM trial failed to demonstrate the beneficial impact of Candesartan in HFpEF. In patients with sinus rhythm some evidence was found for nebivolol, digoxin, spironolactone and candesartan to reduce HF hospitalization [12-14]. High heterogeneity of the enrolled population in randomized clinical trials in $\mathrm{HFpEF}$ is the most frequent reason for failure.
Among elderly patients with HFpEF, several cardiovascular (atrial fibrillation, arterial hypertension, coronary artery disease) and non-cardiovascular diseases (diabetes, obesity, anemia, chronic kidney disease, chronic obstructive pulmonary disease, dementia) significantly impact the quality of life and the outcome of this complex population. Although there is no established strategy for frail patients with $\mathrm{HFpEF}$, a multidisciplinary approach, including also various types of muscular training and nutritional intervention, may provide beneficial effects [15].

The failure of previous trials could be due to the classification of HF by EF, which is easy and fast to perform but does not portray HF to the full. EF does not give the possibility to specify the underlying pathophysiological mechanism and cause of HF syndrome and may lead to an incomplete phenotyping of HF patients. For instance, LV remodeling in HF patients with hypertrophic phenotype shows a different process compared to a dilated phenotype as demonstrated by pressure-volume loops [16]. A potential approach to select therapeutic interventions is to match HFpEF phenotypes on the basis of clinical clusters and biological characteristics. The history of previous myocardial infarction could positively influence the impact of beta-blocker therapy on clinical outcomes. Likewise, patients with HF and metabolic syndrome may benefit from glycemic control, weight loss, and the strict control of the other risk factors [17] . A potential approach to a symptom or phenotype guided therapy is summarized in Table 1. Recently, new data lend support to the strategy of phenotyping HFpEF patients using a biomarker approach. As an example, only in patients with high ratio of serum levels of carboxy-terminal telopeptide of collagen type I to serum matrix metalloproteinase-1 (CITP:MMP-1, an inverse index of myocardial collagen cross-linking), treatment with spironolactone reduced the myocardial collagen content and improved diastolic function. These findings demonstrate the efficacy of spironolactone to reduce myocardial fibrosis and improve LV diastolic function through the stabilization of the collagen fiber in HFpEF patients [18].

Finally, new perspectives are growing with the implementation of new devices such as the CardioMems. In the CHAMPION trial, hemodynamically guided management of patients with HFpEF reduced decompensation leading to hospitalization compared with standard HF management strategies [19].

Table 1. Therapeutical strategies for HFpEF: symptoms, phenotypes and treatment selection.

\begin{tabular}{|c|c|c|c|c|c|c|c|c|c|}
\hline \multirow[t]{3}{*}{ Clinical scenarios } & \multicolumn{9}{|c|}{ Treatment strategies } \\
\hline & & & & & Drugs & & Mete & olism modu & ation \\
\hline & ACEi & ARBs & BBs & MRAs & $\begin{array}{c}\text { ARNI } \begin{array}{c}\mathrm{Na}+\text { channel } \\
\text { blockers }\end{array}\end{array}$ & Nitrates cGMP/PDE5i & Lifestyle & $\begin{array}{l}\text { Invasive } \\
\text { strategies }\end{array}$ & $\begin{array}{c}\text { Glycemic } \\
\text { control }\end{array}$ \\
\hline
\end{tabular}

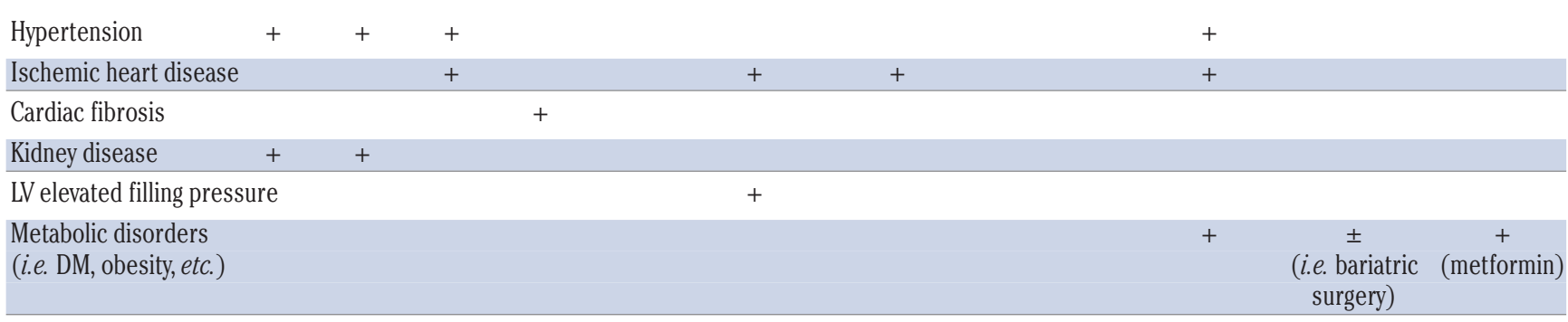

$\mathrm{PH} \pm$ right heart disease

$\pm$

$+$

ACEi, Angiotensin-converting enzyme inhibitor; ARBs, aldosterone receptor blockers; BBs, beta blocker; MRA, mineralocorticoid receptor antagonist; ARNi, angiotensin receptor-neprilysin inhibitor; cGMP, cyclic guanosine monophosphate; PDE5i, phosphodiesterase-5 inhibitor; LV, left ventricle; DM, diabetes mellitus; PH, pulmonary hypertension. 


\section{The safety of traditional drugs in HF and evidence from real world data}

The increasing evidence that demonstrates the net clinical benefit of medical treatment with ACE inhibitors, ARBs, MRAs and beta-blockers has led to the incorporation of these drugs into clinical practice guidelines [20]. Despite all, however, real-world evidence provides findings that are different from those derived from randomized clinical trials. In the elderly population with HFpEF the aim of therapy may change in the presence of co-morbidities such as cancer or dementia. In particular, the treatment of $\mathrm{HFpEF}$ is much more challenging in patients with cognitive impairment. A growing body of evidence, suggests that recommendations are only seldom applied to daily clinical practice in full, thus resulting in either an under-prescription of Class IA drugs or in their sub-optimal dosage [21,22]. When choosing the appropriate therapy, especially in HFrEF patients, special attention should be given to drug contra-indications or situations where specialist advice should be sought. For instance, the use ACEi in patients with pre-existing renal failure [serum creatinine ( $\mathrm{SCr}$ ) levels $>1.4 \mathrm{mg} / \mathrm{dl}$ ] has been linked to a five times higher risk of developing hyperkalemia than those with normal renal function [23]. Moreover, spironolactone has been associated with higher prevalence of renal failure and hyperkalemia, compared to data derived from clinical trials when administered in patients $>70$ years [24]. Likewise, elevated plasma levels of digoxin associated with clinical toxicity, (especially when $>1.2 \mathrm{ng} / \mathrm{ml}$ in AF patients [25]) are a common example of adverse reactions in the elderly population with HF, chronic kidney disease and low body weight. Thus, guidelines recommend that, in patients with $\mathrm{SCr}$ $>2.5 \mathrm{mg} / \mathrm{dl}$ or hyperkalemia $>5.0 \mathrm{mmol} / \mathrm{L}, \mathrm{ACEi}, \mathrm{MRAs}$ and Digoxin should be avoided. As a result of age being a critical parameter in HF therapy, evidence suggests that in the aged population it may be important to closely monitor renal and liver function and fluctuations, body weight trends, electrolytes and polypharmacy to decrease risks related to iatrogenic injury. Drug interactions may occur at the level of drug metabolism, in particular by activation or inhibition of the cytochrome P450 system (e.g., by amiodarone, oral anticoagulation, phenytoin and antibacterials) and/or via inhibition of the P-glycoprotein membrane transporter system (e.g., by amiodarone or digoxin).

As the ratio of drugs/patient increases, the prevalence of undesired drug adverse effects increases exponentially and may beget, or worsen, HF; for instance, several drugs currently prescribed for chronic diseases in the elderly [corticosteroids, NSAIDs including selective cyclo-oxygenase (COX)-2 inhibitors, calcium channel antagonists and thiazolidinediones] are associated with fluid retention and exacerbation of HF. Moreover, other drugs (e.g., class I antiarrhythmic drugs, carbamazepine, tricyclic antidepressants and verapamil) harbour negative inotropic effects. In elderly patients, heart rate may be reduced as result of sick sinus syndrome or atrioventricular block, and baroreceptor function impairment as well as orthostatic dysregulation of blood pressure are often observed [26].

One last note should be dedicated to compliance to medical therapy. In this complex HF landscape, age (young vs old), comorbidities and depression, the number of drugs, socio-economical state and social support are the main reasons for poor medication adherence. In this scenario, the use of a polypill strategy may improve the adherence to therapy and outcome in HF [27].

\section{Conclusions}

HFpEF is a rapidly growing disorder among older persons and could be defined as a geriatric syndrome influenced by aging and affecting all organ systems, embracing varieties of phenotypes. This could explain why clinical trials have often failed to support any specific treatment for this condition. Adoption of a phenotypespecific approach could thus be a key element to successful management. Pharmacological drugs targeting age-related dysfunction, comorbidities, inflammation and oxidative stress may be effective to improve quality of life and mortality in HFpEF. However, we should bear in mind that drug management in elderly patients requires careful monitoring and adjustments to therapy in line with altered pharmacokinetics and pharmacodynamics consideration.

\section{References}

1. Gheorghiade M, Abraham WT, Albert NM, et al. Systolic blood pressure at admission, clinical characteristics, and outcomes in patients hospitalized with acute heart failure. JAMA 2006;296:2217-26.

2. Aurigemma GP, Gottdiener JS, Shemanski L, et al. Predictive value of systolic and diastolic function for incident congestive heart failure in the elderly: the cardiovascular health study. J Am Coll Cardiol 2001;37:1042-8.

3. Covinsky KE, Pierluissi E, Johnston CB. Hospitalization-associated disability: "She was probably able to ambulate, but I'm not sure". JAMA 2011;306:1782-93.

4. Upadhya B, Kitzman DW. Heart Failure with preserved ejection fraction in older adults. Heart Fail Clin 2017;13:485-502.

5. Steinberg BA, Zhao X, Heidenreich PA, et al. Trends in patients hospitalized with heart failure and preserved left ventricular ejection fraction: prevalence, therapies, and outcomes. Circulation 2012;126:65-75.

6. Watson CJ, Gupta SK, O'Connell E, et al. MicroRNA signatures differentiate preserved from reduced ejection fraction heart failure. Eur J Heart Fail 2015;17:405-15.

7. Rech M, Barandiaran Aizpurua A, van Empel V, et al. Pathophysiological understanding of HFpEF: microRNAs as part of the puzzle. Cardiovasc Res 2018;114:782-93.

8. Otto CM. Heartbeat: Is there any effective therapy for heart failure with preserved ejection fraction? Heart 2018;104:361-2.

9. Zheng SL, Chan FT, Nabeebaccus AA, et al. Drug treatment effects on outcomes in heart failure with preserved ejection fraction: a systematic review and meta-analysis. Heart 2018;104:407-15.

10. Cleland JGF, Tendera M, Adamus J, et al.The perindopril in elderly people with chronic heart failure (PEP-CHF) study. Eur Heart J 2006;27):2338-45.

11. Massie BM, Carson PE, McMurray JJ, et al. Irbesartan in patients with heart failure and preserved ejection fraction. $\mathrm{N}$ Engl J Med 2008;359:2456-67.

12. Yusuf S, Pfeffer MA, Swedberg K, et al. Effects of candesartan in patients with chronic heart failure and preserved left-ventricular ejection fraction: the CHARM-Preserved Trial. Lancet 2003;362:777-81.

13. Mulder BA, van Veldhuisen DJ, Crijns HJGM, et al. Effect of nebivolol on outcome in elderly patients with heart failure and atrial fibrillation: insights from SENIORS. Eur J Heart Fail 2012;14:1171-8 
14. Ahmed A, Rich MW, Fleg JL, et al. Effects of digoxin on morbidity and mortality in diastolic heart failure: the ancillary digitalis investigation group trial. Circulation 2006;114:397-403.

15. Kinugasa Y, Yamamoto K. The challenge of frailty and sarcopenia in heart failure with preserved ejection fraction. Heart 2017;103:184-9.

16. Konstam MA, Abboud FM. Ejection fraction: misunderstood and overrated (changing the paradigm in categorizing heart failure). Circulation 2017;135:717-9.

17. Senni M, Paulus WJ, Gavazzi A, et al. New strategies for heart failure with preserved ejection fraction: the importance of targeted therapies for heart failure phenotypes. Eur Heart J 2014;35:2797-815.

18. Ravassa S, Trippel T, Bach D, et al. Biomarker-based phenotyping of myocardial fibrosis identifies patients with heart failure with preserved ejection fraction resistant to the beneficial effects of spironolactone: results from the Aldo-DHF trial. Eur J Heart Fail 2018;20:1290-9.

19. Adamson PB, Abraham WT, Bourge RC, et al. Wireless pulmonary artery pressure monitoring guides management to reduce decompensation in heart failure with preserved ejection fraction. Circ Heart Fail 2014;7:935-44.

20. Ponikowski P, Voors AA, Anker SD, et al. 2016 ESC Guidelines for the diagnosis and treatment of acute and chronic heart failure: The Task Force for the diagnosis and treatment of acute and chronic heart failure of the European Society of Cardiology (ESC)Developed with the special contribution of the Heart Failure Association (HFA) of the ESC. Eur Heart J 2016;37:2129-200.

21. Maggioni AP, Orso F, Calabria S, et al. The real-world evidence of heart failure: findings from 41413 patients of the ARNO database. Eur J Heart Fail 2016;18:402-10.

22. Maggioni AP, Anker SD, Dahlstrom U, et al. Are hospitalized or ambulatory patients with heart failure treated in accordance with European Society of Cardiology guidelines? Evidence from 12,440 patients of the ESC Heart Failure Long-Term Registry. Eur J Heart Fail 2013;15:1173-84.

23. Ahmed A. Use of angiotensin-converting enzyme inhibitors in patients with heart failure and renal insufficiency: how concerned should we be by the rise in serum creatinine? J Am Geriatr Soc 2002;50:1297-300.

24. Svensson M, Gustafsson F, Galatius S, et al. How prevalent is hyperkalemia and renal dysfunction during treatment with spironolactone in patients with congestive heart failure? J Card Fail 2004;10:297-303.

25. Lopes RD, Rordorf R, De Ferrari GM, et al. Digoxin and mortality in patients with atrial fibrillation. J Am Coll Cardiol 2018;71:1063-74.

26. Leibundgut G, Pfisterer M, Brunner-La Rocca H-P. Drug treatment of chronic heart failure in the elderly. Drugs Aging 2007;24:991-1006.

27. Castellano JM, Sanz G, Penalvo JL, et al. A polypill strategy to improve adherence: results from the FOCUS project. J Am Coll Cardiol 2014;64:2071-82. 